\title{
7-axis arm for NDT of surfaces with complex \& unknown geometry
}

\author{
T.P. Sattar, A.A. Brenner \\ Department of Electrical, Computer and Communications Engineering, \\ London South Bank University, 103 Borough Road, London SE1 0AA, \\ UK. Sattartp@Lsbu.ac.uk
}

\begin{abstract}
The aim of the work described here is to design a dexterous and intelligent robotic arm to automate the non-destructive testing and evaluation of geometrically complex industrial products. The robot is required to scan contoured surfaces with ultrasonic and eddy current sensors without the need to position the test pieces precisely, thereby reducing inspection times. In addition, the arm should be lightweight so that it can be carried by wallclimbing and walking robots but at the same time it should be rugged enough to be operated in industrial environments where the tool (sensor probe) comes into contact with rigid and unknown test surfaces. To realise these aims, a seven-axis lightweight and rugged robot arm has been specially developed for non-destructive testing (NDT) and is reported here. The arm weighs $22 \mathrm{~kg}$ and is designed for portability, dexterity, ruggedness in an industrial environment and $1 \mathrm{~mm}$ repeatability. Its NDT payload is $4 \mathrm{~kg}$. Current work is directed at developing a control system for the arm with a proven operating system with further work required to make the arm adapt to uncertain surfaces.
\end{abstract}

Keywords: 7-Axis Arm, Scanning Arm, NDT Robot Arm

\section{Introduction}

Progress in the automation of Non-Destructive Testing (NDT) for both rapid inspection of complex pieces and for remote inspection in hazardous environments requires the development of robotic scanning systems that are dexterous, lightweight, easily transportable from site to site and that can deploy sensor probes on uncertain surfaces with the required probe stand-off or contact force. 
The above characteristics provide the flexibility to use the same scanning arm to provide NDT in various industrial inspection applications thereby reducing instrumentation and tool costs. A lightweight and rugged arm that can be mounted on different types of mobile robots including walking and wall-climbing robots provides the flexibility to scan vertical and large surfaces that are located remotely e.g. ship hulls, petrochemical storage tanks, bridges and tall buildings. Applications of 6-axis arms for inspection invariably use rails to move the along weld lines e.g. the NOMAD project [1] For greater flexibility it would be necessary to use mobile robots to carry the scanning arm. Our arm mounted on different wall climbers would give inspection capability e.g. weld inspection on hulls of cargo container ship [2]. A scanning arm with dexterity and flexibility, approaching that of a human arm, and the ability to adaptively scan complex surfaces that are not precisely known will open up areas of applications in various hazardous or dangerous environments such as nuclear power plant, off-shore oil platforms, large bridges, hulls of ships, oil and chemical storage tanks where the work is difficult for the human operator.

The remote location of a test site where the robotic system will operate, also usually means that the location and geometry of a test surface is not known precisely with the resultant danger that the scanning arm could apply too much or too little surface contact force resulting respectively in damage to the arm/surface/NDT probe or loss of measurement data. There are different kinds of approaches for the recognition of the surface. The problem is the need first to measure the surface and then to control the tool to follow the surface outline. There are several pure spatial approaches.[3] to measure the surface. Our approach is to perform adaptive force control by using a force sensor.

\section{The robotic system}

The robotic system comprises of three main components; a mechanical arm and its AC drive motors, 7 servo amplifiers and a controller. The scanning arm is called the ANSALDO arm. It is interfaced with a Adept control system. The robot arm has 7 revolute joints and a force sensor mounted in its wrist to enable the adaptive control of probe contact forces. The ANSALDO robot arm is controlled by the 9-axis MV-10A Adept motion controller, which provides the possibility to use the DenavitHartenberg algorithm kinematics model to manipulate the robot. The controller and the operator's PC/Laptop have a server-client relationship. The operator can access and control the Adept controller via the Adept Win- 
dows software and delegate the tasks to the robot easily. The motion controllers are interfaced via three interface panels to the servo drives. These provide the connections for the motor brake signals, motor shaft encoder signals, over travel and drive enable and drive error signals from the 7 motors and their corresponding servo drives.

\section{The robot arm}

The main difficulty in designing the seven axis arm was to get the required accuracy of spatial position repeatability and payload of the end effecter while trying to minimise overall mass of the arm.

Also, unlike pick and place robots designed to operate with their base on a flat bench top or floor the arm dynamics must be such as to allow accurate operation with the base in any orientation, as will arise when the arm is transported by the climbing robot vehicles.

The arm is also required to have the dexterity of the human operator with respect to the manipulation of NDT sensors.

Technical specifications of the arm are summarised in Table 1.

- The dexterity of the human operator in manually scanning ultrasonic sensors stems largely from elbow and wrist movements. With 2 elbow joints and 3 wrist joints the robot arm simulates all the key axes of rotation in the human elbow and wrist i.e. all the elbow and joint axes having a large rotation range. However the range of rotation angle about these 5 axes is up to 3 times greater than is possible with the human arm.

- The remaining 2 perpendicular axes (turret and shoulder) are equivalent to one of the waist rotational axes and one of the shoulder axes in the human operator but again with a several fold increase in the range of rotation.

- The arm is comparable to the human arm in the lengths of the upper and lower arm and shorter in waist to shoulder distance. So it is suitable for operation in confined spaces.

- The arm is mounted on the payload platform via 4 bolts equally spaced round the turret base-plate and an adapter plate within a few minutes.

Most of the weight of the arm arises from 7 electric motors. All non-load bearing components such as external covers are made in high grade plastic whilst the arm body is manufactured in lightweight high strength anodised aluminium alloy. Internal gears are made from titanium alloy grade 5.

All joints are controlled by an incremental encoder built into each motor. The arm control system thus records the current position of each joint in a 
non volatile memory. All cables for power, sensor transmission and reception signals and ultrasonic couplant feed are routed through the inside of the arm itself and out through the base of the turret (waist). The arm is made rugged and weatherproofed for a severe environment with anodised aluminium surfaces and sealed and lubricated for life bearings for all joints.

Table 1. Summary of technical specifications of 7-axis arm

\begin{tabular}{|c|l|l|l|}
\hline Joint \# & \multicolumn{1}{|c|}{ Joint Name } & \multicolumn{1}{|c|}{ Joint Speed } & Joint Workspace \\
\hline 1 & Turret & $30^{\circ} / \mathrm{sec}$ & $\pm 170^{\circ}$ \\
\hline 2 & Shoulder & $30^{\circ} / \mathrm{sec}$ & $\pm 170^{\circ}$ \\
\hline 3 & Elbow Roll & $45^{\circ} / \mathrm{sec}$ & $\pm 180^{\circ}$ \\
\hline 4 & Elbow Pitch & $45^{\circ} / \mathrm{sec}$ & $\pm 150^{\circ}$ \\
\hline 5 & Wrist Roll & $90^{\circ} / \mathrm{sec}$ & $\pm 180^{\circ}$ \\
\hline 6 & Wrist Pitch & $90^{\circ} / \mathrm{sec}$ & $\pm 120^{\circ}$ \\
\hline 7 & Hand Roll & $90^{\circ} / \mathrm{sec}$ & $\pm 180^{\circ}$ \\
\hline $\begin{array}{l}\text { Robot Mass }=22 \mathrm{~kg}, \text { Payload }=40 \mathrm{~N}, \text { Arm Length }=300 \mathrm{~mm}, \\
\text { Forearm Length }=300 \mathrm{~mm}, \text { Accuracy }=1 \mathrm{~mm}\end{array}$ \\
\hline
\end{tabular}

The axis of rotation of joint $1,3,5 \& 7$ are perpendicular to the axis of rotation of joint 2, 4, $6 \& 8$. The four characteristic parameters of the robot (two joint and two link parameters) are shown in Table 2. The joint distance $d$, the link length $a$ and the link twist angle $\alpha$ are fixed parameters.

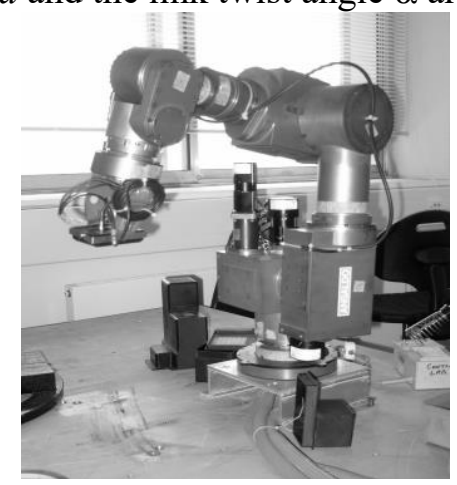

Fig. 1. 7-axis revolute joint ANSALDO arm 
In operation the ANSALDO robot has a repeatability of $1 \mathrm{~mm}$ which is sufficient for ultrasonic and eddy currents probes to find any cracks. The robots servo control is sufficient and provides a good system response, e.g. to a step, as can be seen in figure 2 .

\section{The advantages of the compilation}

The ANSALDO arm provides the dexterity and flexibility approaching that of a human arm whereas the Adept controller enables assembly operations to be performed at higher speeds than are possible with other force sensing units. This is integrated by the use of the AdeptForce package that allows the robot to react to sensed forces and moments. The Adept system has also the advantage to be easily extendable into an automation system with vision guidance and inspection, by use of the AdeptVision Module and software. The high level language $\mathrm{V}+$ implements extensive vision tools for vision-related operations like image capture, enhancement, and analysis. It is a programming language that provides a solution to programming needs in a robotic work cell, including safety, robot motion, vision operations, force sensing and I/O.

The efficient use of system memory and reduction in overall system complexity of the Adept system combined with the ANSALDO robot arm enables a real-time scanning system which permits complex motions to be executed quickly, using the $\mathrm{V}+$ continuous trajectory computation. The system allows on-line program generation and modification and can concurrently interact with an operator. Besides a real-time operating system, the Adept controller also offers advanced servo-control features and the Kinematics Device Modules which result in a powerful control system. We have used the "6/7 Axis Rotary Device Module"[15] which allows V+ to understand the geometry and specifications of a particular robotic mechanism. This software defines the characteristics of a particular 7 axis robot. It is a separate file stored in the system file which implements all the characteristics of the particular robot. Figure 3 shows the ANSALDO robot in its zero position, all the angles are set to zero. It is the graphical representation of the data or knowledge the Adept controller has about the robot it is controlling. This software enables a more structured view and enables the operator to use instructions which can move the robot in a less complicated way. The robot device module defines a robot with seven axes where the third axis is the redundant one. 


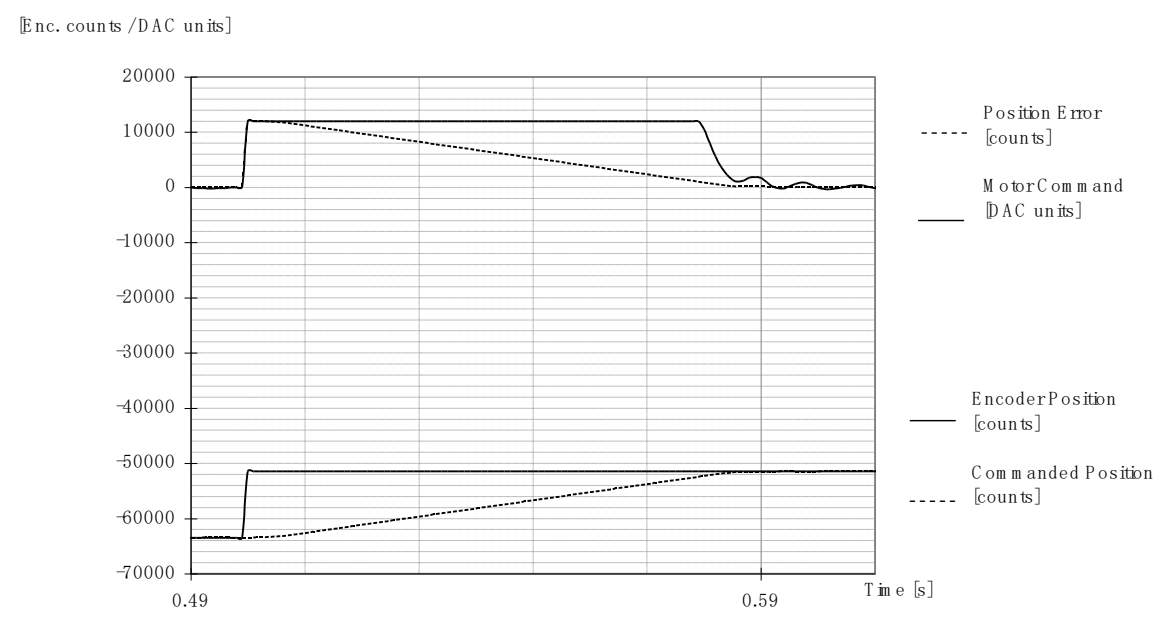

Fig. 2. Step response and error for one joint of tuned system

Table 2. The resulting characteristic table for the revolute ANSALDO arm

\begin{tabular}{|c|c|c|c|c|}
\hline $\begin{array}{l}\text { Axis } \\
\text { (Joint) }\end{array}$ & $\begin{array}{l}\text { Joint angle } \theta \\
{\left[^{\circ}\right] \text { variable }}\end{array}$ & $\begin{array}{l}\text { Joint distance } d \\
{[\mathrm{~mm}] \quad \text { - fixed }} \\
-\end{array}$ & $\begin{array}{l}\text { Link length } \\
a[\mathrm{~mm}] \quad- \\
\text { fixed - }\end{array}$ & $\begin{array}{l}\text { Link twist } \\
\text { angle } \alpha \quad- \\
\text { fixed - }\end{array}$ \\
\hline 1 (Base) & $\theta_{1}$ & 185 & 0 & $-\pi / 2$ \\
\hline $\begin{array}{l}2 \\
\text { (Shoulder) }\end{array}$ & $\theta_{2}$ & 150 & 0 & $\pi / 2$ \\
\hline $\begin{array}{l}3 \\
\text { (Redun- } \\
\text { dant waist) }\end{array}$ & $\theta_{3}$ & 310 & 0 & $\pi / 2$ \\
\hline 4 (Elbow) & $\theta_{4}$ & 80 & 0 & $-\pi / 2$ \\
\hline 5 (Yaw) & $\theta_{5}$ & 300 & 0 & $-\pi / 2$ \\
\hline 6 (Pitch) & $\overline{\theta_{6}}$ & 0 & 0 & $\pi / 2$ \\
\hline 7 (Roll) & $\theta_{7}$ & 260 & 0 & 0 \\
\hline
\end{tabular}




\section{Contact force}

The main difficulty in automating NDT is to establish an accurate adaptation to the test surface (remotely located or of complex shape such as aircraft turbine blades that have a complex surface geometry) to obtain high quality data from possible defects. It is then necessary to respond to surface changes while going over the surface and at the same time to also orientate the NDT probe in the right way and position to get the best results..

A force sensor from JR3 is already mounted on the robot's wrist. This will provide surface adaptation in real time thus allowing task-based control strategies to be employed. Although the most common method to profile a surface is to use vision sensors, force control has advantages when pushing a sensor probe against a surface to maintain a given contact force. Since this is how ultrasound inspection is performed the force sensor for adapting to the unknown surface is a very good option. Other research shows the possibility of a multi-sensor based robot which would further expand this project by the implementation of a visual sensor, like a camera [4]. The Adept Controller provides the possibility to add a force control module to control the contact force in two different modes, guarded and protect mode [5].

The RJ3 sensor (Model: 90M31A) has onboard electronics to provide force and torque data at very high bandwidths and with very low noise. Data for all six axes is returned at a rate of $8 \mathrm{kHz}$. The sensor has a load capacity of minimum $25,50 \mathrm{lbs}$ up to $68,58 \mathrm{lbs}$ [6].

\section{Conclusion}

The ANSALDO robot arm with a weight of $22 \mathrm{~kg}$ is a reasonably lightweight robotic scanning system and therefore easily transportable. It is designed for portability, dexterity, ruggedness in an industrial environment. It is built with rugged gears to withstand possible collisions when working in probe contact mode. A great deal of flexibility is provided to reach a given point inside its workspace and to reach around possible obstacles. The combination of the Adept controller and the 7 axis ANSALDO scanning arm has resulted in a fast and dexterous robot that has a powerful control system which with further additions of force control and vision will allow rapid inspection of complex pieces and inspection on uncertain surfaces located in remote and hazardous environments. In operation the ANSALDO robot has a repeatability of $1 \mathrm{~mm}$ which is sufficient for ultrasonic and eddy currents probes to find most defects. 


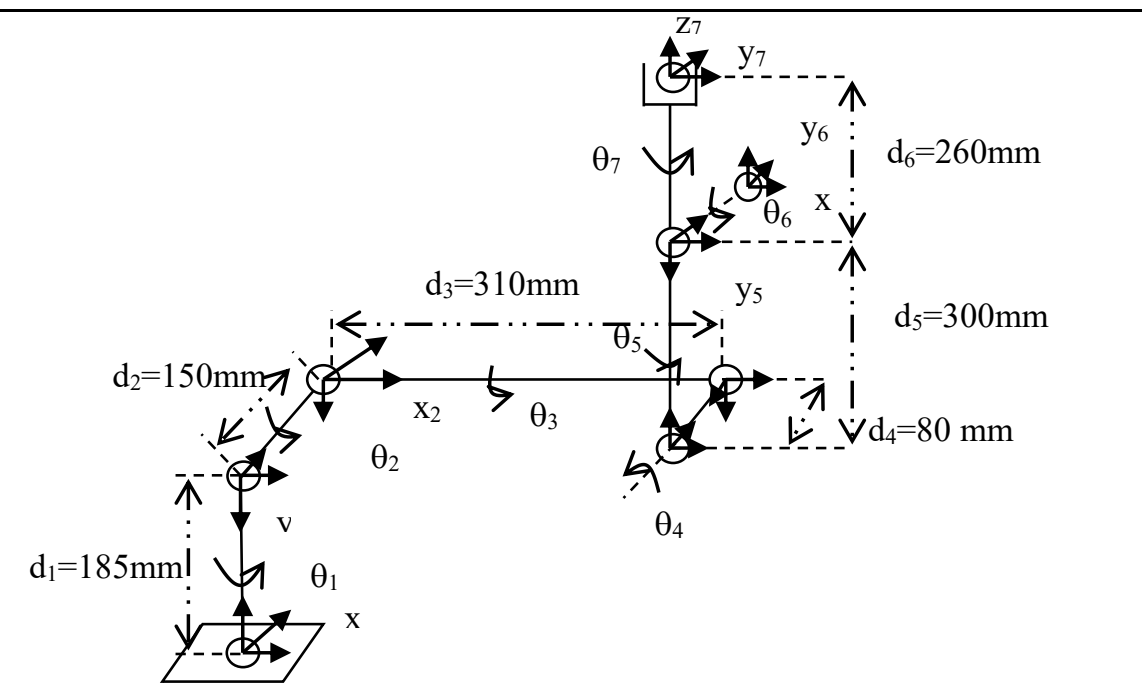

Fig.3. The robot arm (zero position) configuration applied to device module.

\section{Acknowledgements}

The authors gratefully thank the EPSRC (UK) and TWI Ltd (UK) for their funding of Ms Alina-Alexandra Brenner's CASE Award.

\section{References}

1. URL http://www.automation.com/store/pid9244.php [Jun 2005]

2. Sattar T.P., Alaoui M., Chen S., Bridge B. (2001) ROBHULL: A magnetically adhering Wall Climbing Robot to Perform Continuous Welding of Long Seams and Non Destructively Test the Welds on the Hull of a Container Ship, 8th IEEE Conference on Mechatronics and Machine Vision in Practice, M2VIP2001, pp. 408-414, ISBN 962-442-191-9, Paper No. 129.

3. Smith M L (2001): Surface Inspection Techniques, Edmundsbury Press Limited, Suffolk, UK.

4. Xiao D., Ghosh B. K., Xi N, and Tarn T. J (1999): Sensor-based hybrid position/force control of a robot manipulator in an uncalibrated environment. IEEE transactions on control systems technology, Vol.8, No.4, Jul 2000.

5. Adept Technology, Inc.: Adept Force VME User's Guide (1995), USA.

6. URL http://www.jr3.com/dsp.html [Jun 2005]. 